\title{
An Optimized Isolation Network for the Wilkinson Divider
}

\author{
Vahdettin Tas, Student Member, IEEE, and Abdullah Atalar, Fellow, IEEE
}

\begin{abstract}
We propose an isolation network to simultaneously improve the input return loss, output return loss and isolation of the Wilkinson power divider in a wide bandwidth. The required even mode and odd mode reflection coefficients of the isolation network are calculated. Constructed even and odd mode circuits are combined to give the desired isolation network. Analytical expressions for the optimal component values for a single-section divider are given. Compared with the single-section Wilkinson divider, the final design can triple the bandwidth for an input-output return loss and isolation of greater than $25 \mathrm{~dB}$. Broadband characteristic is achieved without increasing the number of sections hence extra length and insertion loss are avoided. Wide operation bandwidth of the new divider is verified by experimental results. The proposed method can be applied to a two-section divider, also broadening its bandwidth.
\end{abstract}

Index Terms - Even mode, isolation network, odd mode, reflection coefficients, wideband power divider, Wilkinson divider.

\section{INTRODUCTION}

$\mathbf{P}$ OWER dividers are used at microwave frequencies to direct the power to two or more loads. Normally, they maintain the matched condition at all ports within the operation band. Moreover, a good isolation between the output ports is desired to eliminate the interaction between the loads. When used in the opposite direction, power dividers can combine power from two or more power sources. The Wilkinson power divider was introduced in 1960 [1]. Around the center frequency it is matched at all ports and isolated at the output ports within a relatively narrow bandwidth. Cohn [2] introduced the multisection hybrids to improve the operation bandwidth of the Wilkinson divider. Broadband power division using multisection structures was revealed in several studies [3]-[8]. Wide operation bandwidth is also achieved by utilizing tapered lines. They are constructed using a continuum of a long tapered line [9] or multisection tapered lines [10]. The main disadvantage of using multi sections is the increase in the length and the insertion loss of the divider. To avoid this, a number of researchers designed reduced size dividers [11]-[14]. Another approach to improve the bandwidth response of the Wilkinson divider aims dual band

Manuscript received June 30, 2014; revised September 22, 2014; accepted October 23, 2014. Date of publication November 14, 2014; date of current version December 02, 2014. This paper is an expanded version from the IEEE MTT-S International Microwave Symposium, Tampa Bay, FL, USA, June 1-6 2014.

The authors are with the Department of Electrical and Electronics Engineering, Bilkent University, Ankara 06800, Turkey (e-mail: vtas@ee.bilkent. edu.tr; aatalar@bilkent.edu.tr).

Color versions of one or more of the figures in this paper are available online at http://ieeexplore.iee.org.

Digital Object Identifier 10.1109/TMTT.2014.2365533 operation. This is achieved by using $R L C$ networks in series or parallel configuration between the output ports [15]-[17], coupled lines [18], parallel stubs along the transmission path [19] and right-left handed transmission lines [20]. These designs also require an increase in length. Other works [21], [22] focus on the isolation network design for isolation bandwidth improvement. Length increment is avoided by modifying only the isolation network. In these works, the isolation bandwidth is widened while the input return loss is kept unchanged. Due to the position of the isolation resistors extra insertion loss is faced.

Our goal is to design an isolation network that improves the bandwidth of input return loss, output return loss and isolation, simultaneously. This way, the number of sections is not increased hence extra length and loss are avoided. We first derive an equation relating the input return loss, output return loss and isolation values of a symmetrical two way power divider. Then we calculate the optimal reflection coefficients of the isolation network in the even mode and odd mode cases. Circuits are constructed to realize the calculated reflection coefficients. The resulting circuits are combined in the final divider structure. Preliminary experimental results of the single-section divider were presented in [23]. Here, detailed analytical work and simulation results for the single and two-section dividers are presented along with new experimental results.

\section{S-PARAMETER Relation FOR A LossLess SYMMETRICAL 2-WAY DIVIDER}

In Fig. 1, a symmetrical multisection 2-way combiner/divider network is depicted. The network is composed of lossless lumped or distributed components apart from the isolation arms shown with gray boxes. The contents of the gray boxes are delineated in Fig. 2. It is composed of symmetrical white boxes with resistors in the middle. The white boxes in Figs. 1 and 2 can have more than one series or shunt lossless components as long as the symmetry between the arms is maintained. S-parameter matrix equation of such a three port is given by

$$
\left[\begin{array}{l}
b_{1} \\
b_{2} \\
b_{3}
\end{array}\right]=\left[\begin{array}{lll}
S_{11} & S_{21} & S_{21} \\
S_{21} & S_{22} & S_{32} \\
S_{21} & S_{32} & S_{22}
\end{array}\right]\left[\begin{array}{l}
a_{1} \\
a_{2} \\
a_{3}
\end{array}\right]
$$

where $\left|a_{n}\right|^{2}$ and $\left|b_{n}\right|^{2}$ are the incident and reflected powers at port $n .\left|S_{21}\right|$ can be expressed as

$$
\left|S_{21}\right|=\sqrt{\frac{1-\left|S_{11}\right|^{2}}{2}}
$$

If $a_{1}=0$ and $a_{2}=a_{3}=1 / \sqrt{2}$ are applied, we find

$$
\left|b_{1}\right|=\sqrt{1-\left|S_{11}\right|^{2}}
$$




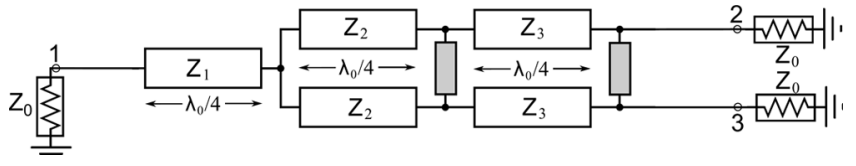

Fig. 1. Symmetrical 2-way power combiner/divider network. White boxes are lossless. Gray boxes represent the lossy isolation arms.

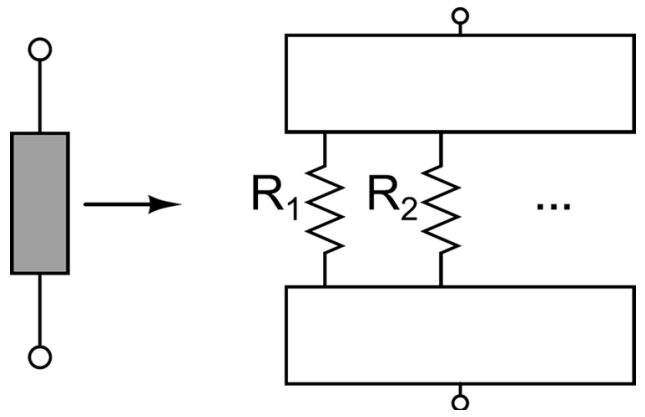

Fig. 2. Possible contents of a gray box: Symmetrical lossless white boxes with isolation resistors in the middle.

$$
b_{2}=b_{3}=\frac{S_{22}+S_{32}}{\sqrt{2}}
$$

The incident power is $\left|a_{2}\right|^{2}+\left|a_{3}\right|^{2}=1$. Due to the even excitation, there is no dissipation in the isolation arms, and the total incident power equals the transmitted power plus the total reflected power:

$$
\left|a_{2}\right|^{2}+\left|a_{3}\right|^{2}=\left|b_{1}\right|^{2}+\left|b_{2}\right|^{2}+\left|b_{3}\right|^{2} .
$$

From (3)-(5) we find

$$
1=\left|b_{1}\right|^{2}+\left|b_{2}\right|^{2}+\left|b_{3}\right|^{2}=\left(1-\left|S_{11}\right|^{2}\right)+\left|S_{22}+S_{32}\right|^{2}
$$

and finally we arrive at the result ${ }^{1}$

$$
\left|S_{11}\right|=\left|S_{22}+S_{32}\right| \text {. }
$$

Corollary 1: For the generic 2-way divider of Fig. 1, if $\mid S_{22}+$ $S_{32} \mid<\delta$ and $\left|S_{22}-S_{32}\right|<\delta$, then $\left|S_{11}\right|<\delta,\left|S_{22}\right|<\delta$ and $\left|S_{32}\right|<\delta$.

Proof: Defining $S_{22}+S_{32}=\delta_{1} e^{j \theta_{1}}$ and $S_{22}-S_{32}=$ $\delta_{2} e^{j \theta_{2}}$, where $\delta_{1}, \delta_{2} \leq \delta$ and $\theta_{1}, \theta_{2}$ are arbitrary angles, we have

$$
\begin{aligned}
& \left|S_{22}\right|=\frac{\left|\delta_{1} e^{j \theta_{1}}+\delta_{2} e^{j \theta_{2}}\right|}{2} \leq \frac{\left(\delta_{1}+\delta_{2}\right)}{2} \leq \delta \\
& \left|S_{32}\right|=\frac{\left|\delta_{1} e^{j \theta_{1}}-\delta_{2} e^{j \theta_{2}}\right|}{2} \leq \frac{\left(\delta_{1}+\delta_{2}\right)}{2} \leq \delta .
\end{aligned}
$$

$\left|S_{11}\right|<\delta$ directly follows from (7).

For the generalized two-way divider in Fig. 1, the excitation $a_{1}=0, a_{2}=1, a_{3}=1$ results in the even mode operation. The S-parameter matrix (1) can be rewritten as

$$
\left[\begin{array}{l}
b_{1} \\
b_{2} \\
b_{3}
\end{array}\right]=\left[\begin{array}{lll}
S_{11} & S_{21} & S_{21} \\
S_{21} & S_{22} & S_{32} \\
S_{21} & S_{32} & S_{22}
\end{array}\right]\left[\begin{array}{l}
0 \\
1 \\
1
\end{array}\right] .
$$

${ }^{1}$ Equation (7) was implicitly stated in [2].
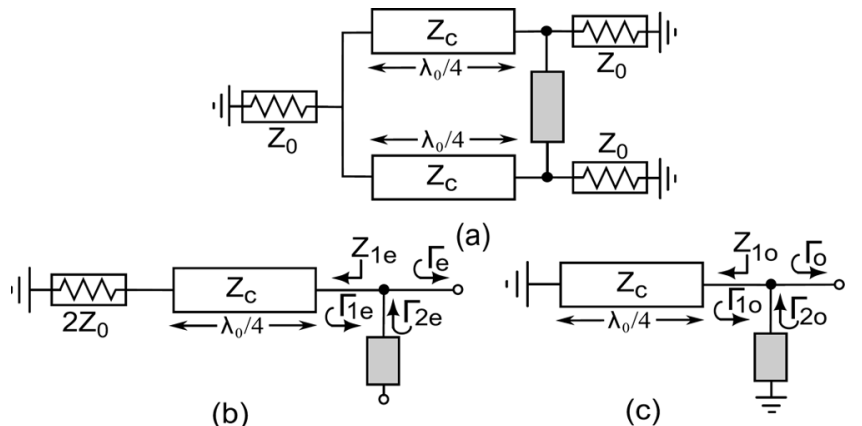

Fig. 3. (a) Single-section Wilkinson divider with a gray box in the isolation arm. (b) Even mode equivalent circuit. c) Odd mode equivalent circuit.

So, the reflection coefficient at the second port equals to

$$
\Gamma_{e}=\frac{b_{2}}{a_{2}}=S_{22}+S_{32} .
$$

Similarly, the excitation $a_{1}=0, a_{2}=1, a_{3}=-1$ results in the odd mode operation. The S-parameter matrix (1) can be rewritten as

$$
\left[\begin{array}{l}
b_{1} \\
b_{2} \\
b_{3}
\end{array}\right]=\left[\begin{array}{lll}
S_{11} & S_{21} & S_{21} \\
S_{21} & S_{22} & S_{32} \\
S_{21} & S_{32} & S_{22}
\end{array}\right]\left[\begin{array}{c}
0 \\
1 \\
-1
\end{array}\right] .
$$

So, the reflection coefficient at the second port equals to

$$
\Gamma_{o}=\frac{b_{2}}{a_{2}}=S_{22}-S_{32} .
$$

$S_{22}+S_{32}$ and $S_{22}-S_{32}$ are the even mode $\left(\Gamma_{e}\right)$ and odd mode $\left(\Gamma_{o}\right)$ reflection coefficients at port 2 . From Corollary 1 we can conclude that keeping the even mode and odd mode reflection coefficients at port 2 below a certain level assures that the input-output return loss and isolation parameters are kept below the same level.

In the literature, broadband operation of the power dividers is generally achieved at the expense of an increase in the length and insertion loss of the dividers. In those designs, the isolation networks are composed of floating components without a ground connection, hence the isolation network does not affect the even mode circuit. Consequently, the input return loss can not be tuned by the isolation network. This is a significant loss of a degree of freedom. By the inclusion of components with a ground connection, the isolation network can be active both in the even mode as well as in the odd mode. With such an approach both $S_{22}-S_{32}$ and $S_{22}+S_{32}$ can be tuned by the isolation network.

\section{Optimal Choice of Isolation Network IN THE EVEN AND ODD MODES}

Our goal is to find an optimal isolation network for the singlesection Wilkinson divider depicted in Fig. 3(a). The isolation network is represented with a gray box. $Z_{c}$ can be different than $\sqrt{2} Z_{0}$ for generality. Fig. 3(b) and (c) shows the even and odd mode equivalent circuits with the gray box open-circuited and shorted at its mid point, respectively. 


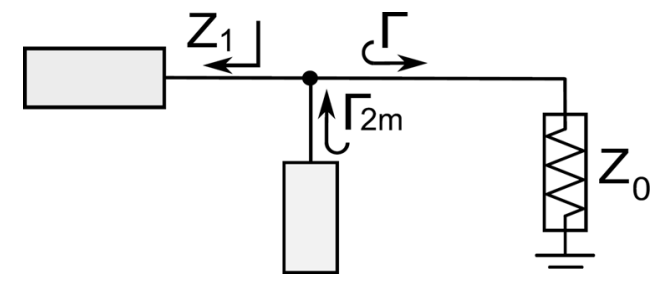

Fig. 4. Two networks connected in parallel. One has an input impedance of $Z_{1}$, the other one has an input reflection coefficient of $\Gamma_{2 m}$ to give $\Gamma=0$.

For a parallel connection of two one-port networks shown in Fig. 4 , the perfect match condition $(\Gamma=0)$ requires

$$
\Gamma_{2 m}=\frac{1}{2 \bar{Z}_{1}-1} \text { for } \bar{Z}_{1} \neq 0
$$

where $\bar{Z}_{1}=Z_{1} / Z_{0}$ is the normalized input impedance of the first block and $\Gamma_{2 m}$ is the input reflection coefficient of the second block giving the perfect match.

The normalized impedances $\bar{Z}_{1 e}$ and $\bar{Z}_{1 o}$ defined in Fig. 3 are equal to

$$
\begin{aligned}
& \bar{Z}_{1 e}=\frac{Z_{1 e}}{Z_{0}}=\frac{2+j \bar{Z}_{c} \tan \left(\frac{\pi f}{2 f_{0}}\right)}{1+j \frac{2}{\bar{Z}_{c}} \tan \left(\frac{\pi f}{2 f_{0}}\right)} \\
& \bar{Z}_{1 o}=\frac{Z_{1 o}}{Z_{0}}=j \bar{Z}_{c} \tan \left(\frac{\pi f}{2 f_{0}}\right)
\end{aligned}
$$

where $f_{0}\left(=w_{0} / 2 \pi\right)$ is the center frequency and $\bar{Z}_{c}=Z_{c} / Z_{0}$. The reflection coefficients $\Gamma_{2 e m}$ and $\Gamma_{2 o m}$ of the even and odd mode isolation circuits that will generate a perfect match can be calculated using (14), (15), and (16):

$$
\begin{aligned}
\Gamma_{2 e m} & =\frac{1+j \frac{2}{\bar{Z}_{c}} \tan \left(\frac{\pi f}{2 f_{0}}\right)}{3+j\left(2 \bar{Z}_{c}-\frac{2}{\bar{Z}_{c}}\right) \tan \left(\frac{\pi f}{2 f_{0}}\right)} \\
\Gamma_{2 o m} & =\frac{1}{j 2 \bar{Z}_{c} \tan \left(\frac{\pi f}{2 f_{0}}\right)-1} .
\end{aligned}
$$

\section{A. Even Mode Isolation Circuit}

The trajectory of $\Gamma_{2 e m}$ is drawn on the Smith chart of Fig. 5 for $\bar{Z}_{c}=0.95 \sqrt{2}, \sqrt{2}$ and $1.05 \sqrt{2}$. Even mode isolation circuit must be lossless, otherwise the insertion loss of the divider will increase. So, its reflection coefficient must lie on the unity circle of the Smith chart. $\Gamma_{2 e m}$ characteristic can be approximated with a shorted quarter-wave stub of impedance $Z_{p}$ as in Fig. 6. The corresponding $\Gamma_{2 e}$ is depicted by dashed lines in Fig. 5. For $\bar{Z}_{c}=\sqrt{2}, \Gamma_{2 e}$ and $\Gamma_{2 e m}$ coincide only at the center frequency and deviate fast with frequency. For $\bar{Z}_{c}>\sqrt{2}$, the situation is even worse. With $\bar{Z}_{c}<\sqrt{2}, \Gamma_{2 e}$, and $\Gamma_{2 e m}$ coincide at two frequencies and the highest deviation occurs at the center frequency, $f_{0}$, and at band edges. This characteristic resembles a second degree Chebyshev response maximizing the bandwidth. So, we choose $\bar{Z}_{c}<\sqrt{2}$ to have a wideband approximation. The deviation at $f_{0}$ can be adjusted by the value of $Z_{c}$. At $f_{0}$, the shorted quarter-wave stub $\left(Z_{p}\right)$ acts like an open-circuit. Using Corollary-1 we aim to satisfy $\left|\Gamma_{e}\right| \leq \delta$. The deviation is max-

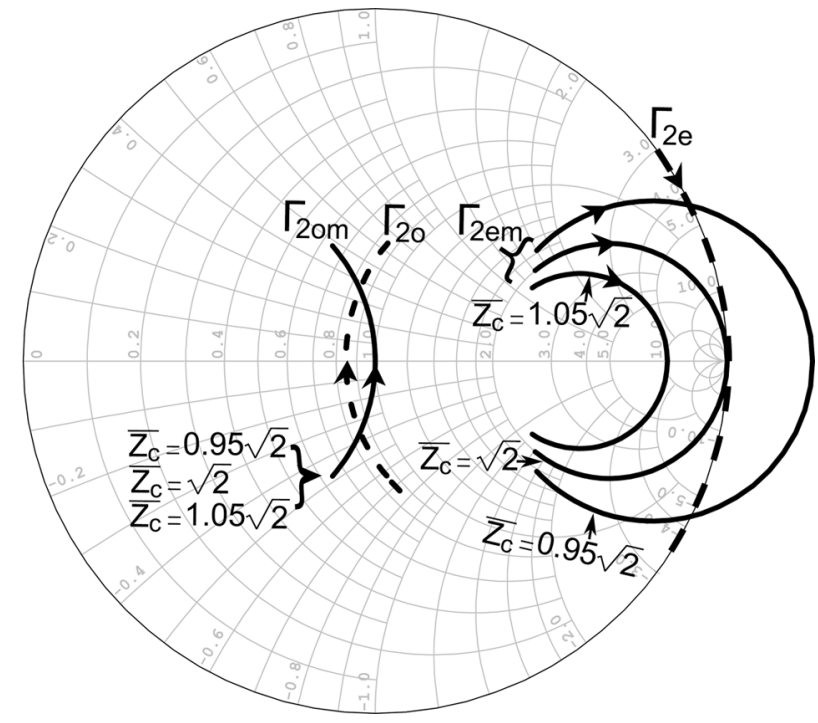

Fig. 5. $\Gamma_{2 e m}$ and $\Gamma_{2 o m}$ on the Smith chart (solid lines) as the frequency is swept from $f_{0} / 2$ to $3 f_{0} / 2$ (in the direction of arrows) for $\bar{Z}_{c}=0.95 \sqrt{2}, \sqrt{2}$ and $1.05 \sqrt{2}$. Approximations, $\Gamma_{2 e}$ and $\Gamma_{2 o}$, (dashed lines) are also shown.

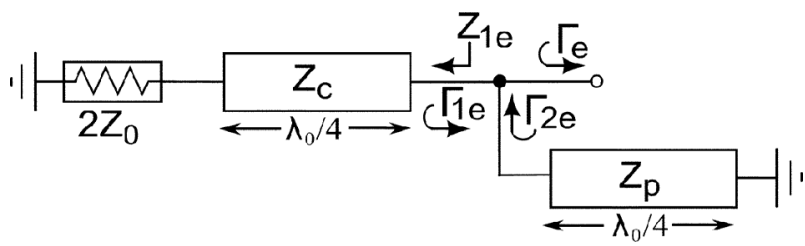

Fig. 6. Even mode equivalent circuit with the shorted quarter-wave stub of impedance $Z_{p}$ at the isolation arm.

imum at $f_{0}$, so $\left|\Gamma_{e}\left(f_{0}\right)\right|=\delta$. Referring to Fig. 6 , since $\Gamma_{e}=\Gamma_{1 e}$ at $f_{0}$, the value of $Z_{1 e}\left(f_{0}\right)$ is equal to

$$
Z_{1 e}\left(f_{0}\right)=\frac{Z_{c}^{2}}{2 Z_{0}}=Z_{0} \frac{1-\delta}{1+\delta}
$$

Hence, we have

$$
\bar{Z}_{c}=\sqrt{\frac{2(1-\delta)}{1+\delta}} \approx \sqrt{2}(1-\delta) .
$$

To calculate the bandwidth and the optimal value of $Z_{p}$, we refer to the Smith chart shown in Fig. 7. The goal is to bend the $Z_{1 e}$ trajectory into the $\left|\Gamma_{e}\right|=\delta$ circle using the shorted transmission line with the characteristic impedance of $Z_{p}$. The lowest normalized conductance on the locus of the $\left|\Gamma_{e}\right|=\delta$ circle is $(1-\delta) /(1+\delta)$. So, the frequencies at which $Z_{1 e}$ results a conductance of $(1-\delta) /(1+\delta)$ are the minimum and the maximum frequencies satisfying the $\left|\Gamma_{e}\right| \leq \delta$ condition. The lower and upper frequencies are marked as $f_{1}$ and $f_{2}$, respectively, in Fig. 7.

At $f=f_{1}$, we have:

$$
\operatorname{Re}\left\{\frac{1}{\bar{Z}_{1 e}\left(f_{1}\right)}\right\}=\frac{1-\delta}{1+\delta} .
$$

Using (15) and (20), (21) can be expanded as

$$
\operatorname{Re}\left\{\frac{\sqrt{2}(1-\delta)+j 2 \tan \left(\frac{\pi f_{1}}{2 f_{0}}\right)}{2(1-\delta)\left[\sqrt{2}+j(1-\delta) \tan \left(\frac{\pi f_{1}}{2 f_{0}}\right)\right]}\right\}=\frac{1-\delta}{1+\delta} .
$$




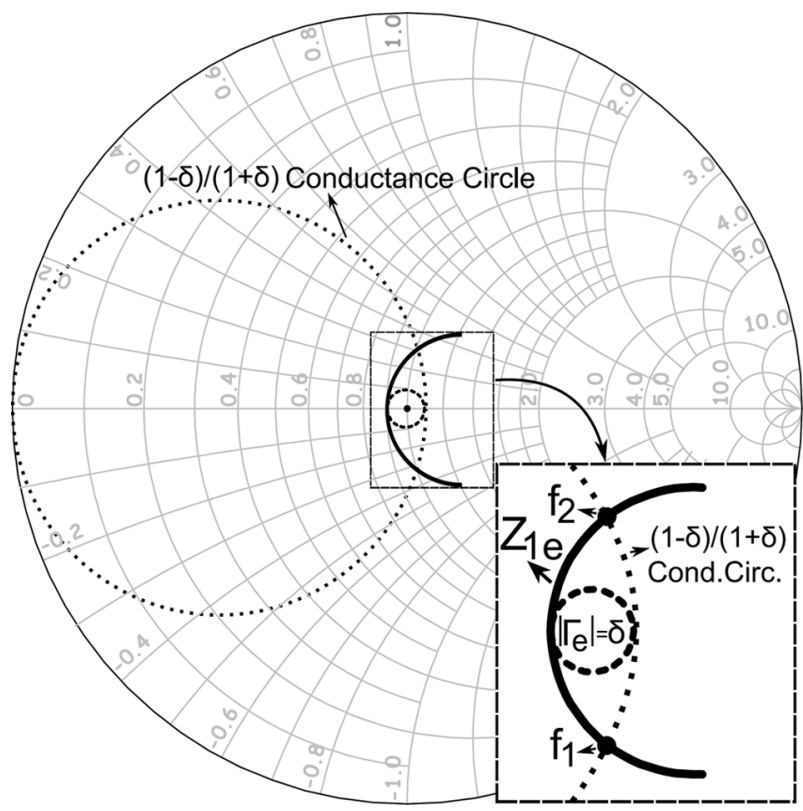

Fig. 7. Trajectories of the $(1-\delta) /(1+\delta)$ constant conductance circle and $\left|\Gamma_{e}\right|=\delta$ circle along with the trajectory of $Z_{1 e}$ (shown in Fig. 6).

We find

$$
\tan \left(\frac{\pi f_{1}}{2 f_{0}}\right)=\sqrt{\frac{1-3 \delta}{4 \delta-3 \delta^{2}+\delta^{3}}}
$$

For small $\delta$, we can ignore the $\delta^{3}$ term, and write $f_{1}$ as

$$
\frac{f_{1}}{f_{0}} \approx \frac{2}{\pi} \tan ^{-1} \sqrt{\frac{1-3 \delta}{4 \delta-3 \delta^{2}}}
$$

We note that $f_{2}-f_{0}=f_{0}-f_{1}$. For example, for $\delta=0.1(-20 \mathrm{~dB}), f_{1}=0.6 f_{0}$ and $f_{2}=1.4 f_{0}$.

The value of $Z_{p}$ is calculated by considering that the imaginary part of the $1 / Z_{1 e}$ admittance should be compensated perfectly at $f_{1}$ :

$$
\operatorname{Im}\left\{\frac{1}{\bar{Z}_{1 e}}\right\}=\frac{1}{\bar{Z}_{p} \tan \left(\frac{\pi f_{1}}{2 f_{0}}\right)} .
$$

So, we have

$$
\operatorname{Im}\left\{\frac{\sqrt{2}(1-\delta)+j 2 \tan \left(\frac{\pi f_{1}}{2 f_{0}}\right)}{2(1-\delta)\left[\sqrt{2}+j(1-\delta) \tan \left(\frac{\pi f_{1}}{2 f_{0}}\right)\right]}\right\}=\frac{1}{\bar{Z}_{p} \tan \left(\frac{\pi f_{1}}{2 f_{0}}\right)} .
$$

Using (24) the value of $\bar{Z}_{p}$ is extracted from (26) as

$$
\bar{Z}_{p} \approx \sqrt{2} \frac{1+2 \delta-2 \delta^{2}}{1-\delta-7 \delta^{2}}
$$

$\bar{Z}_{p}$ approaches to $\sqrt{2}$ as $\delta$ gets closer to zero. With $\delta=0.1$, (27) results in $\bar{Z}_{p}=2.0$

If a lumped circuit is preferred, this shorted $\lambda / 4$ line can be approximated as a parallel $L C$ circuit [24] with the component values expressed as

$$
w_{0} L_{p}=\frac{4 Z_{p}}{\pi} \quad \text { and } \quad L_{p} C_{p}=\frac{1}{w_{0}^{2}} .
$$

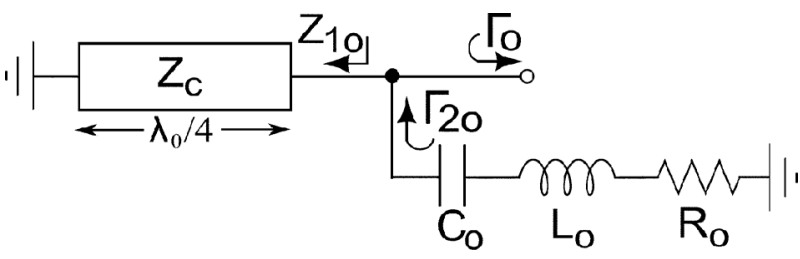

Fig. 8. Odd mode equivalent circuit with the optimal isolation network. $L_{o}$ and $C_{o}$ are resonant at the center frequency $f_{0}$.

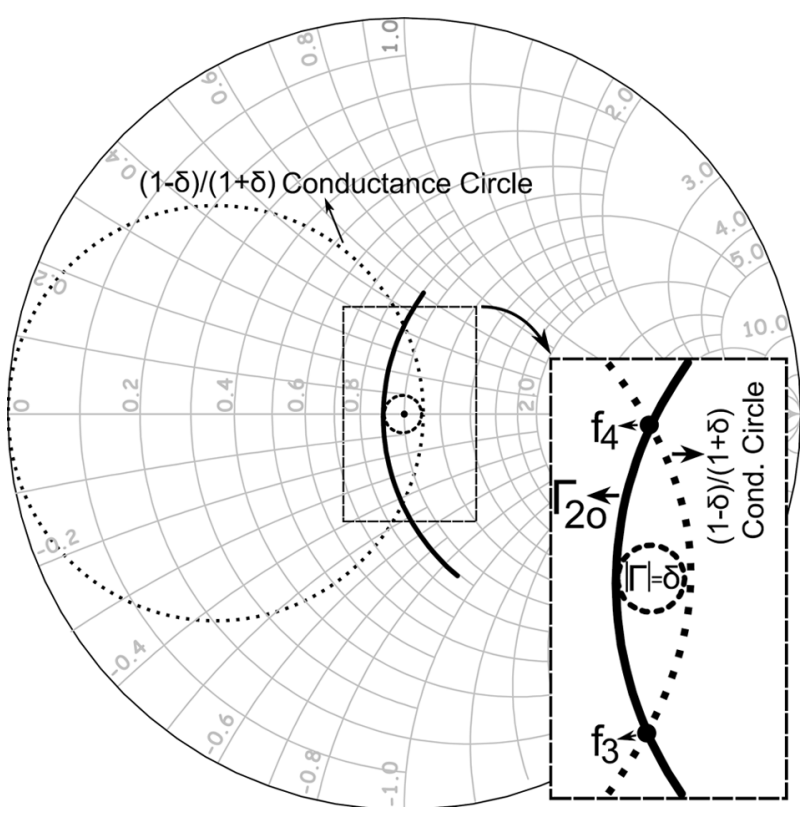

Fig. 9. Trajectories of the $(1-\delta) /(1+\delta)$ constant conductance circle and $|\Gamma|=\delta$ circle along with the trajectory of $\Gamma_{2 o}$ of Fig. 8.

\section{B. Odd Mode Isolation Circuit}

Smith chart trajectories of $\Gamma_{2 o m}$ for three different $Z_{c}$ values (which are almost equal to each other) are shown in Fig. 5. The locus of $\Gamma_{2 o m}$ follows a constant conductance circle. A series $R L C$ circuit resonant at $f_{0}$ shown in Fig. 8 can be utilized to approximate the desired characteristics.

The same approach as the even mode case is followed to calculate the component values of the odd mode isolation network analytically. Referring to Fig. $8, \Gamma_{2 o}$ is the reflection coefficient of the series $R_{o} L_{o} C_{o}$ network. The goal is to maximize the bandwidth of the $\left|\Gamma_{o}\right| \leq \delta$ condition. Fig. 9 shows the $|\Gamma|=\delta$ circle on the Smith chart together with the trajectory of $\Gamma_{2 o}$. The points marked as $f_{3}$ and $f_{4}$ represent the edge frequencies that $\Gamma_{2 o}$ can be fitted into the $\Gamma=\delta$ circle by the shorted stub of impedance $Z_{c}$.

The impedance of a series $L C$ resonator is not symmetric around the center frequency. We have $f_{4}-f_{0}>f_{0}-f_{3}$. Therefore, $2\left(f_{0}-f_{3}\right)$ is the bandwidth of the $\left|\Gamma_{o}\right| \leq \delta$ condition. For the circuit in Fig. 8 at $f_{0}, C_{o}$ resonates with $L_{o}$ and the quarter-wave stub $Z_{c}$ is open-circuit. We have $\Gamma_{o}\left(f_{0}\right)=$ $\Gamma_{2 o}\left(f_{0}\right)=\left(R_{o}-Z_{0}\right) /\left(R_{o}+Z_{0}\right)$. As shown in Fig. $9, \Gamma_{2 o}\left(f_{0}\right)=$ $-\delta$. So, the value of the resistor is equal to

$$
\bar{R}_{o}=\frac{R_{o}}{Z_{0}}=\frac{1-\delta}{1+\delta} .
$$




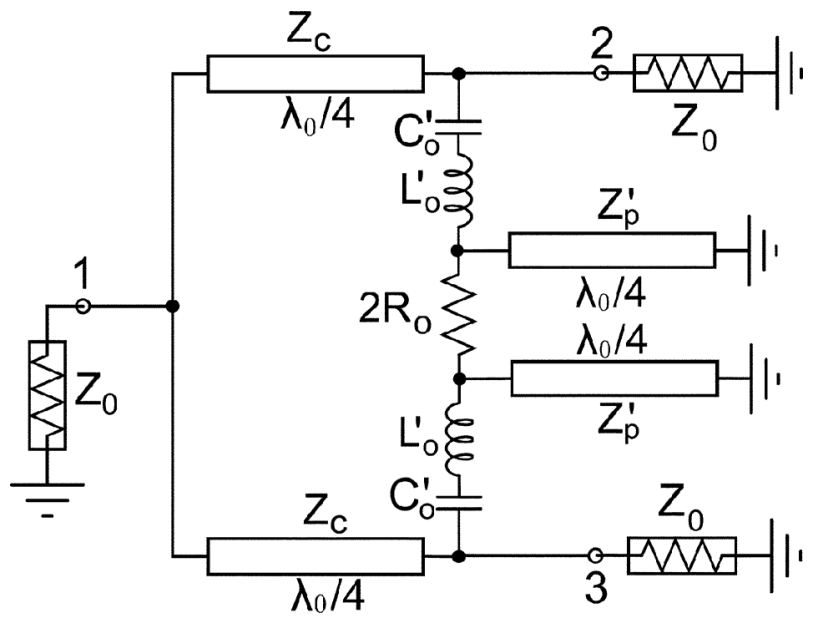

Fig. 10. Schematic diagram of the divider by merging the optimal even mode and odd mode isolation networks.

$L_{o}$ can be calculated by imposing two conditions at $f=f_{3}$. $\Gamma_{2 o}$ trajectory crosses the $(1-\delta) /(1+\delta)$ conductance circle and the transmission line with the impedance of $Z_{c}$ compensates the reactive part of $\Gamma_{2 o}$ perfectly. So, we have two equations:

$$
\begin{aligned}
& \operatorname{Re}\left\{\frac{Z_{0}}{R_{o}-j 2 \pi f_{3} L_{o}\left(\frac{f_{0}^{2}}{f_{3}^{2}}-1\right)}\right\}=\frac{1-\delta}{1+\delta} \\
& \operatorname{Im}\left\{\frac{Z_{0}}{R_{o}-j 2 \pi f_{3} L_{o}\left(\frac{f_{0}^{2}}{f_{3}^{2}}-1\right)}\right\}=\frac{1}{\bar{Z}_{c} \tan \left(\frac{\pi f_{3}}{2 f_{0}}\right)} .
\end{aligned}
$$

Using (29) and (30) we find

$$
2 \pi f_{3} \frac{L_{o}}{Z_{0}}\left(\frac{f_{0}^{2}}{f_{3}^{2}}-1\right)=\frac{2 \sqrt{\delta}}{1+\delta}
$$

Inserting (32) in the denominator of (31) and using (20), $f_{3}$ is calculated as

$$
\frac{f_{3}}{f_{0}}=\frac{2}{\pi} \tan ^{-1}\left(\frac{(1+\delta)^{3 / 2}}{\sqrt{8 \delta(1-\delta)}}\right) .
$$

Equation (33) together with (32) result in

$$
\frac{w_{0} L_{o}}{Z_{0}}=\frac{2 \sqrt{\delta}}{1+\delta} \frac{\frac{f_{0}}{f_{3}}}{\left(\frac{f_{0}}{f_{3}}\right)^{2}-1} .
$$

For example, with $Z_{0}=50 \Omega$ and $\delta=0.1$, we find $f_{3}=$ $0.596 f_{0}$ and $L_{o}=4.23 \mathrm{nH}$. We note that the odd mode bandwidth is greater than the even mode bandwidth.

\section{Combining the Even And Odd Mode ISOLATION NETWORKS}

We merge the isolation circuits of Figs. 6 and 8 as shown in Fig. 10. Fig. 11 depicts the approximations involved in the merging process. Due to the interaction between the even and odd mode networks, some component values are shown with a prime symbol to indicate a possible modification in the calculated component values of the previous section.

The value of $Z_{c}$ was determined in the even mode circuit by the condition at $f_{0}$. Since the isolation network does not affect

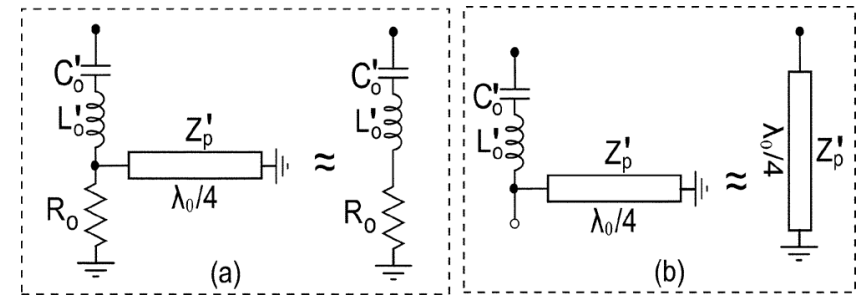

Fig. 11. Equivalent representation of the isolation network for the divider in Fig. 10. (a) Odd-mode. (b) Even-mode.
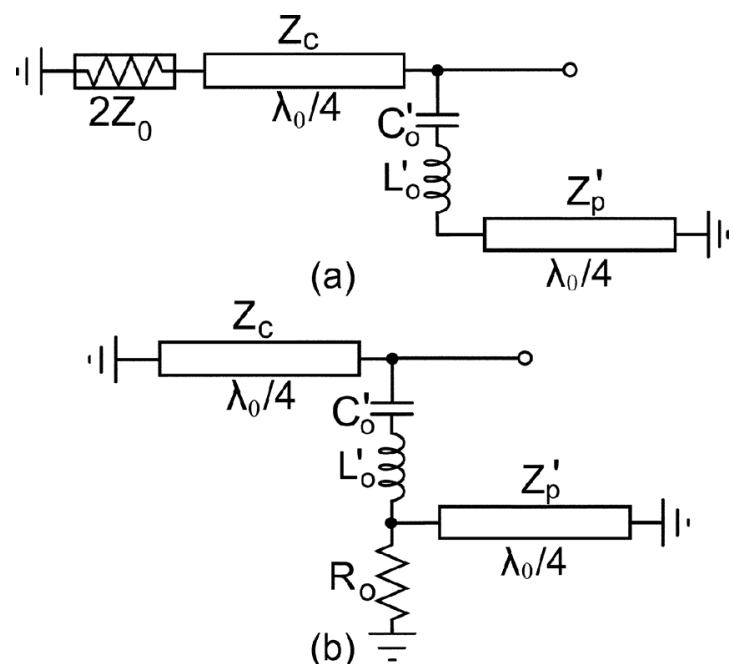

Fig. 12. Even (upper) and odd (lower) mode equivalent circuits of the divider in Fig. 10.

the even mode circuit at $f_{0}, Z_{c}$ value of (20) will not be modified. Similarly, $R_{o}$ was determined in the odd mode circuit by the condition at $f_{0}$. Since $Z_{p}{ }^{\prime}$ is open circuit at $f_{0}$, the value of $R_{0}$ given in (29) will not change.

The new values of $Z_{p}{ }^{\prime}$ and $L_{0}{ }^{\prime}$ can be calculated following the analysis of the previous sections. Fig. 12 shows the even and odd mode equivalent circuits of the divider. Referring to Fig. 12(a), (26) is modified due to the presence of $L_{o}{ }^{\prime}$ and $C_{o}{ }^{\prime}$ :

$$
\begin{array}{r}
\operatorname{Im}\left\{\frac{\sqrt{2}(1-\delta)+j 2 \tan \left(\frac{\pi f_{1}}{2 f_{0}}\right)}{2(1-\delta)\left[\sqrt{2}+j(1-\delta) \tan \left(\frac{\pi f_{1}}{2 f_{0}}\right)\right]}\right\}= \\
=\frac{Z_{0}}{Z_{P}^{\prime} \tan \left(\frac{\pi f_{1}}{2 f_{0}}\right)-2 \pi f_{1} L_{o}^{\prime}\left(\frac{f_{0}^{2}}{f_{1}^{2}}-1\right)}
\end{array}
$$

where $f_{1}$ is expressed in (24). $f_{1}$ is independent of the problem handled here, it is determined solely by $Z_{c}$.

From Fig. 12(b), (30) and (31) are modified to

$\operatorname{Re}\left\{\frac{Z_{0}}{R_{o} \| j Z_{p}^{\prime} \tan \left(\frac{\pi f_{3}^{\prime}}{2 f_{0}}\right)-j 2 \pi f_{3}^{\prime} L_{o}^{\prime}\left(\frac{f_{0}^{2}}{f_{3}^{\prime 2}}-1\right)}\right\}=\frac{1-\delta}{1+\delta}$

$\operatorname{Im}\left\{\frac{Z_{0}}{R_{o} \mid j Z_{p}^{\prime} \tan \left(\frac{\pi f_{3}^{\prime}}{2 f_{0}}\right)-j 2 \pi f_{3}^{\prime} L_{o}^{\prime}\left(\frac{f_{0}^{2}}{f_{3}^{\prime 2}}-1\right)}\right\}=\frac{Z_{0}}{Z_{c} \tan \left(\frac{\pi f_{3}^{\prime}}{2 f_{0}}\right)}$.

$f_{3}^{\prime}$ is used instead of the $f_{3}$ in (33) because it is dependent on $Z_{p}^{\prime}$ and $L_{o}^{\prime}$. A simultaneous solution of (35), (36), and (37) to find $f_{3}^{\prime}, Z_{p}^{\prime}$ and $L_{o}^{\prime}$ is not possible. To find the values, the mean square 
TABLE I

NORMALIZED COMPONENT VALUES FOR INPUT-OUTPUT RETURN LOSSES AND ISOLATION BETTER THAN $\delta(\mathrm{dB})$.

\begin{tabular}{|c|c|c|c|c|c|}
\hline$\delta(d B)$ & $\Delta f / f_{0}$ & $\bar{Z}_{c}$ & $2 \bar{R}_{o}$ & ${\overline{w_{0} L_{o}}}^{\prime}$ & $\bar{Z}_{p}^{\prime}$ \\
\hline \hline-20 & 0.80 & 1.28 & 1.63 & 0.64 & 2.41 \\
\hline-25 & 0.60 & 1.34 & 1.79 & 0.84 & 1.98 \\
\hline-30 & 0.45 & 1.37 & 1.88 & 0.96 & 1.73 \\
\hline-35 & 0.34 & 1.39 & 1.93 & 1.02 & 1.59 \\
\hline-40 & 0.25 & 1.4 & 1.96 & 1.06 & 1.51 \\
\hline
\end{tabular}

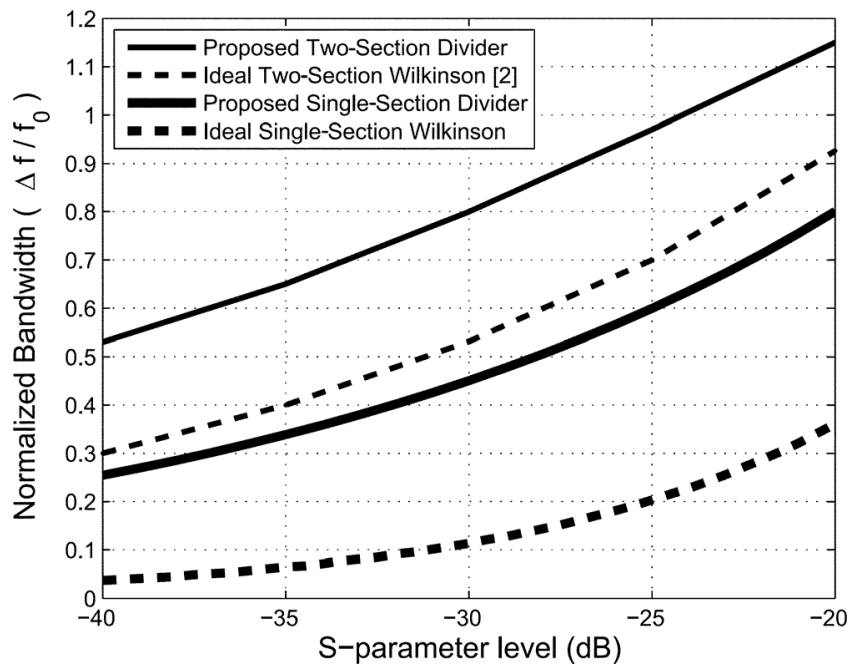

Fig. 13. Normalized bandwidth comparison of the prosed divider structure with the classical single-section Wilkinson divider and Cohn's design of twosection Wilkinson divider [2].

error in these equations are minimized numerically. Results of the numerical calculations are used to express $Z_{p}^{\prime}$ and $L_{o}^{\prime}$ by the following approximations

$$
\begin{aligned}
\bar{Z}_{p}^{\prime} & =\frac{Z_{p}^{\prime}}{Z_{0}} \approx \sqrt{2}+10 \delta \\
{\overline{w_{0}} L_{o}^{\prime}}^{\prime} & =\frac{w_{0} L_{o}^{\prime}}{Z_{0}} \approx 1.1-4.6 \delta .
\end{aligned}
$$

Table I lists the results normalized with $Z_{0}$. The achievable normalized bandwidth and required component values for the corresponding $\delta$ level are listed. The divider bandwidth, $\Delta f$, is limited by the bandwidth of the even mode circuit: $\Delta f=$ $2\left(f_{0}-f_{1}\right) . Z_{c}, f_{1}, R_{o}, Z_{p}^{\prime}$, and $L_{o}^{\prime}$ are determined from (20), (24), (29), (38), and (39), respectively. $C_{o}^{\prime}$ is resonant with $L_{o}^{\prime}$ at $f_{0}$. Our results are verified using a microwave circuit simulator. ${ }^{2}$

It is possible to apply the proposed method to a two-section divider. Using a similar isolation arm for both sections, it is possible to extend the bandwidth considerably. The results for the two-section case are given in Appendix A.

Fig. 13 compares the bandwidth capabilities of the proposed single-section and two-section dividers with the classical singlesection Wilkinson divider and Cohn's two-section Wilkinson divider [2]. A significant bandwidth improvement is achieved. For $25 \mathrm{~dB}$ of input-output return loss and isolation, three times wider bandwidth is obtained in comparison to a single-section Wilkinson. The bandwidth of a two-section Wilkinson divider

\footnotetext{
${ }^{2}$ AWR Corp. El Segundo, CA 90245, USA, http://www.awrcorp.com
}

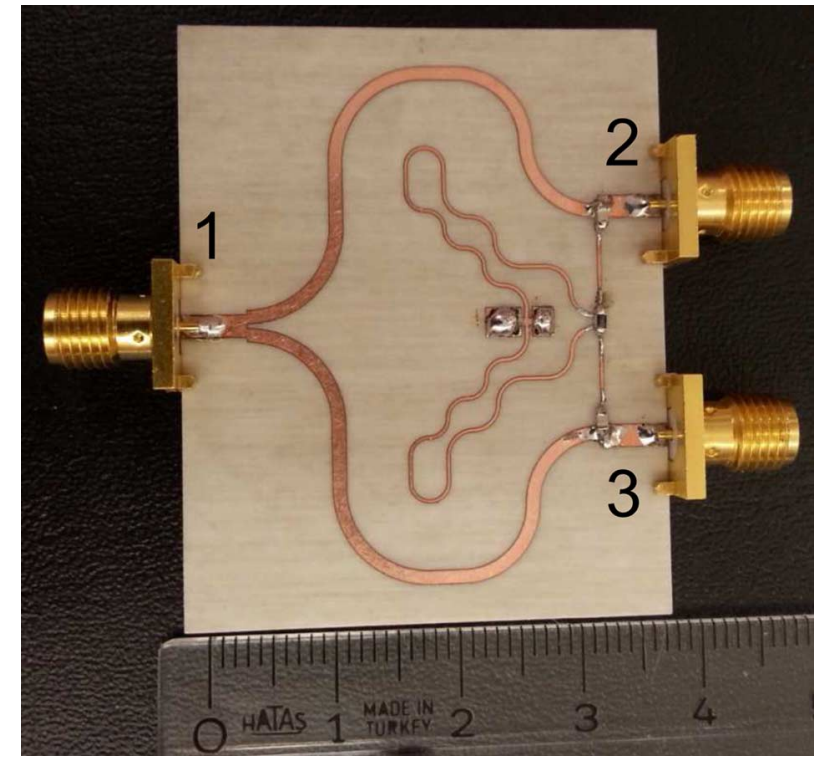

Fig. 14. Implemented 2-way power divider of Fig. 10.

is almost reached with a single-section divider, avoiding the extra size and insertion loss of the two-section divider.

In terms of insertion loss, the proposed divider is similar to a single-section Wilkinson divider, because the input signal propagates a distance of exactly one quarter wavelength. In fact, our divider is more advantageous in terms of the loss, since the impedance transforming line has a characteristic impedance smaller than $\sqrt{2} Z_{0}$ resulting in a wider line width. Considering the size of the divider, the $\lambda_{0} / 4$ line in the isolation arm is shorted at one end and it has a high characteristic impedance with a narrow width. This enables one to use layout techniques such as bending or meandering of the line to get a compact size. We note that a two-section Wilkinson divider with two isolation resistors cannot be shaped in the same manner to reduce the size.

We note that presence of a ground path in the isolation network generates nulls in $S_{21}$. These frequencies can be calculated by considering the sum of the impedances of the $Z_{p}^{\prime}$ line and $L_{o}^{\prime} C_{o}^{\prime}$ pair:

$$
Z_{p}^{\prime} \tan \left(\frac{\pi f}{2 f_{0}}\right)-2 \pi f L_{o}^{\prime}\left(\frac{f_{0}^{2}}{f^{2}}-1\right)=0
$$

Using (38) and (39), (40) can be rewritten as

$$
(\sqrt{2}+10 \delta) \tan \left(\frac{\pi f}{2 f_{0}}\right)=(1.1-4.6 \delta) \frac{f}{f_{0}}\left(\frac{f_{0}^{2}}{f^{2}}-1\right) .
$$

For example, for $\delta=0.1(-20 \mathrm{~dB}),(41)$ is satisfied at two frequencies, at $0.36 f_{0}$ and $1.8 f_{0}$, outside the operation bandwidth $\left(0.6 f_{0}-1.4 f_{0}\right)$ of the divider.

\section{Simulation AND Experimental Results}

The proposed divider in Fig. 10 is implemented for experimental verification at a center frequency of $f_{0}=1 \mathrm{GHz} . \delta=$ 


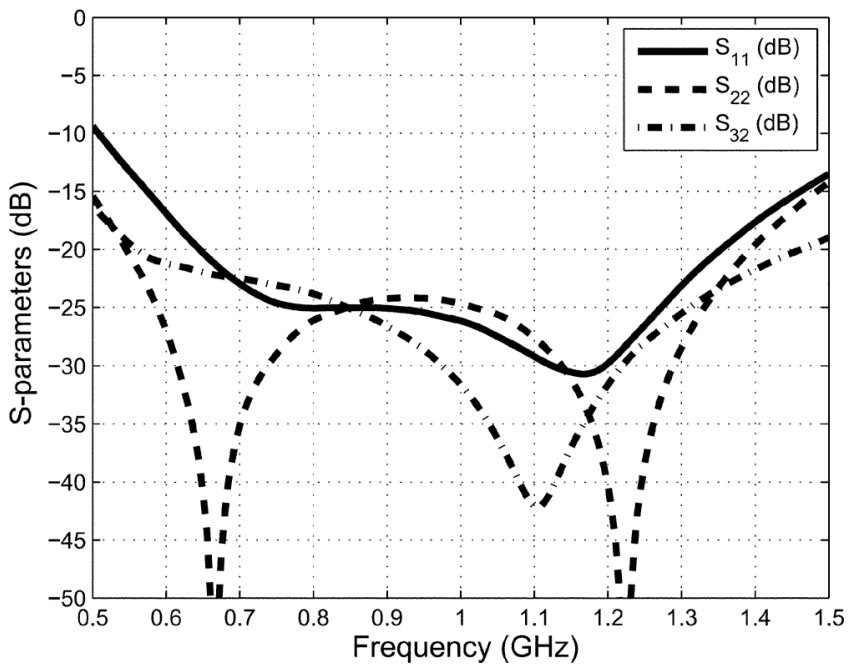

Fig. 15. Simulated S-parameter characteristics of the divider in Fig. 14.

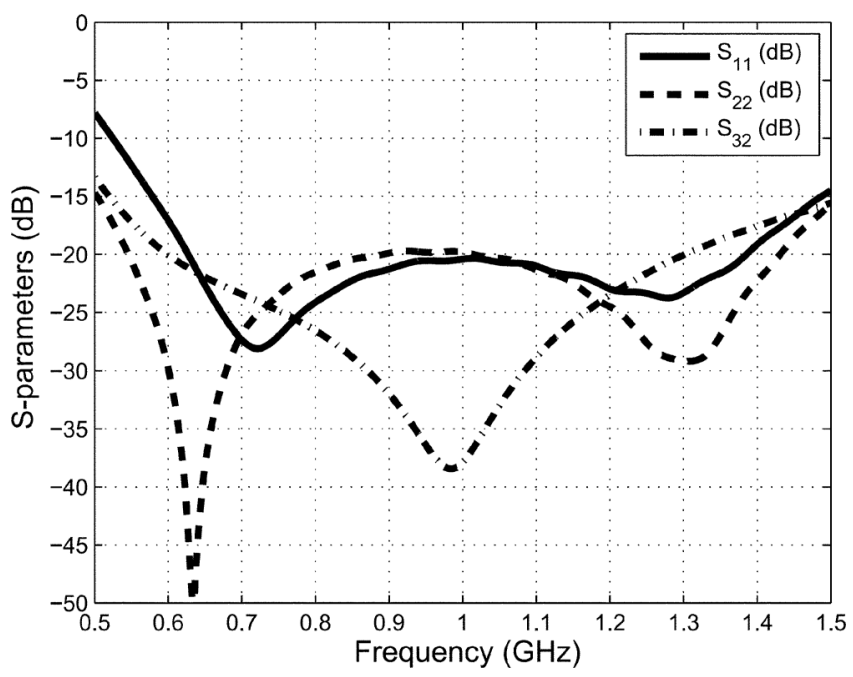

Fig. 16. Measured S-parameter characteristics of the divider in Fig. 14.
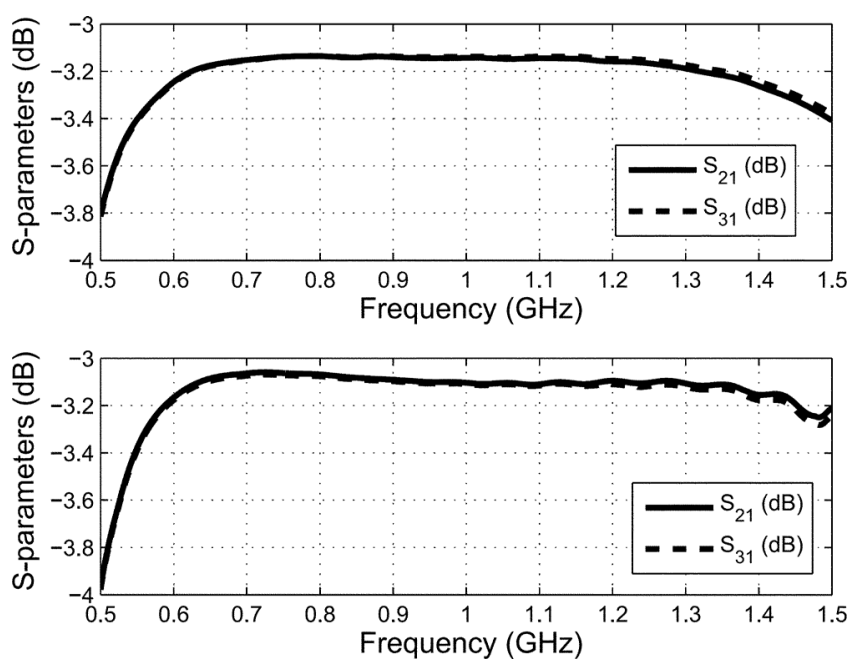

Fig. 17. Simulated (upper) and measured (lower) S-parameter characteristics of the divider in Fig. 14.

$-20 \mathrm{~dB}$ is the design goal. As shown in Table I, the theoretical fractional bandwidth is $\Delta f / f_{0}=0.8$ implying a 20 -dB bandwidth of $0.6 \mathrm{GHz}$ to $1.4 \mathrm{GHz}$. The divider is simulated using

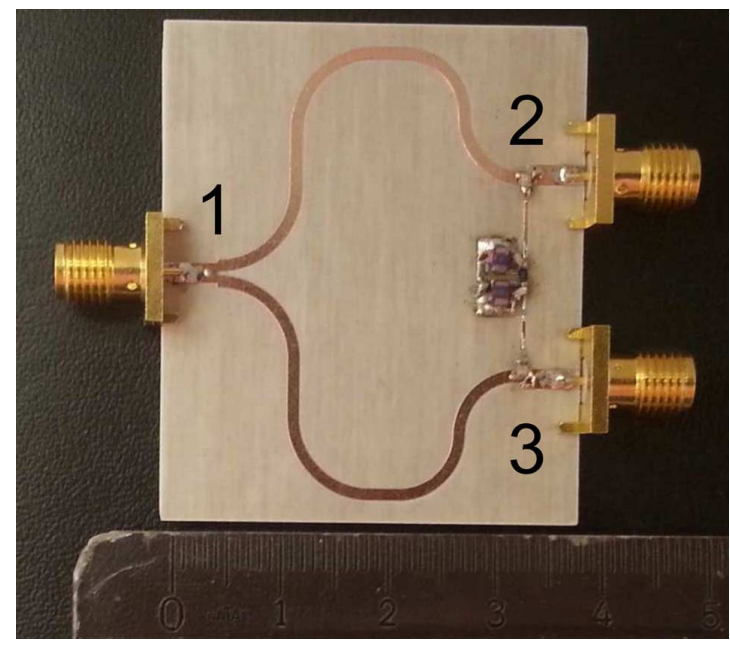

Fig. 18. Modified version of divider in Fig. 14 upon the replacement of the shorted $\lambda_{0} / 4$ lines with the parallel $L C$ resonators.

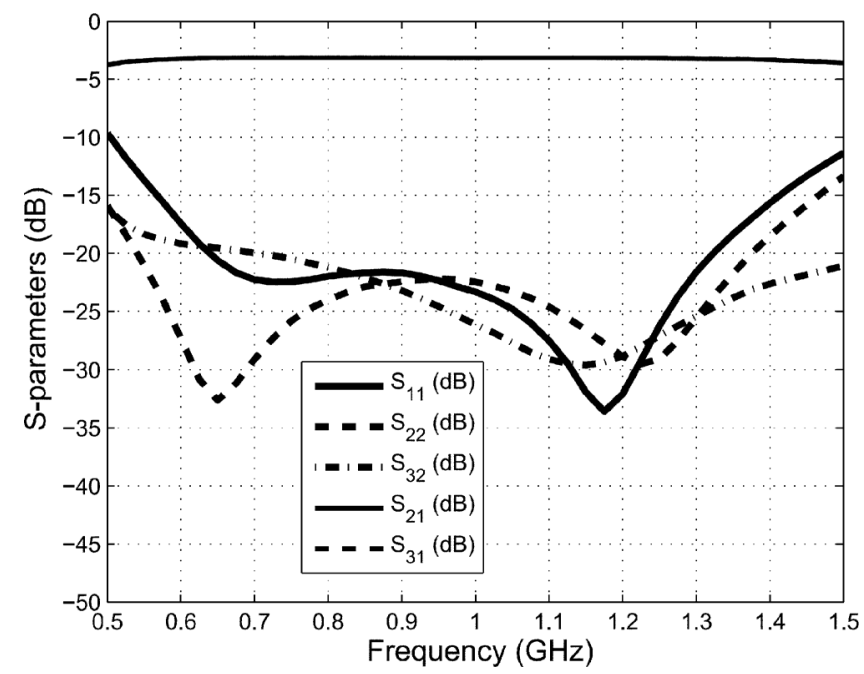

Fig. 19. Simulated response of the divider in Fig. 18.

the microwave circuit simulator and an electromagnetic simulator. ${ }^{3}$ With ideal components, $\Delta f / f_{0}=0.8$ is achieved using the values given in Table I. Inclusion of the junction effects and component parasitics result in a reduction of the bandwidth. To alleviate these effects, the component values are tuned slightly.

Fig. 14 shows the photo of the divider implemented on a RO4003 ${ }^{4}$ substrate with a thickness of $0.8 \mathrm{~mm}$. $L_{o}$ inductors are implemented using high impedance microstrip lines of 0.3 $\mathrm{mm}$ in width and $6.7 \mathrm{~mm}$ in length. Presence of $L_{o}$ in the isolation circuit provides a natural separation between ports 2 and 3 , reducing the length of subsequent connections and improving the isolation between the output ports [25], [26]. In the divider of Fig. 14, the width of $Z_{c}$ lines are $1.1 \mathrm{~mm}$ corresponding to a characteristic impedance of $66 \Omega$. $Z_{p}$ lines have a width of 0.3 $\mathrm{mm}$ resulting in an impedance of $116 \Omega .0603$ package 5.6-pF capacitors and a $75 \Omega$ resistor are used. Figs. 15 and 17 show the simulation results of the electromagnetic simulator. The output return loss and the isolation are better than $20 \mathrm{~dB}$ in the targeted

${ }^{3}$ SONNET Software, North Syracuse NY 13212, USA, http://www.sonnetsoftware.com

${ }^{4}$ Rogers Corp. Rogers, CT 06263, USA, http://www.rogerscorp.com 


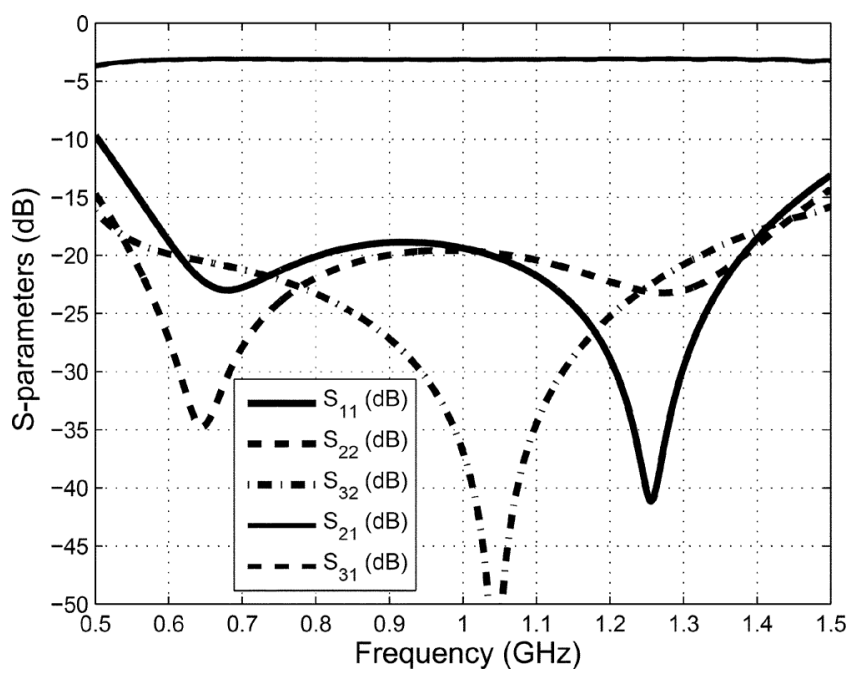

Fig. 20. Measured response of the divider in Fig. 18.

TABLE II

COMPARISON TABLE

\begin{tabular}{|c|c|c|c|c|}
\hline Reference & Technique & Size & $\begin{array}{l}\text { Normalized } \\
\text { Bandwidth }\end{array}$ & $\begin{array}{l}\text { Loss } \\
(\mathrm{dB})\end{array}$ \\
\hline [5] & $\begin{array}{l}\text { Microcoaxial } \\
\text { Multisection }\end{array}$ & $6 \lambda_{0} / 4$ & $20-\mathrm{dB}: 1.10$ & 1 \\
\hline [27] & $\begin{array}{l}\text { Multi Wafer } \\
\text { Multisection }\end{array}$ & $\lambda_{0}$ & $15-\mathrm{dB}: 1.15$ & 1 \\
\hline [10] & $\begin{array}{c}\text { Tapered } \\
\text { Multisection }\end{array}$ & $5 \lambda_{0} / 6$ & $15-\mathrm{dB}: 1.60$ & 0.3 \\
\hline [7] & $\begin{array}{c}\text { Three-section } \\
\text { Coplanar Waveguide }\end{array}$ & $3 \lambda_{0} / 4$ & 15-dB: 1.15 & 0.8 \\
\hline [12] & $\begin{array}{l}\text { Lumped } \\
\text { Inductors }\end{array}$ & $\lambda_{0} / 10$ & $20-d B: 0.15$ & 0.3 \\
\hline [28] & $\begin{array}{l}\text { Right-Left Handed } \\
\text { isolation elements }\end{array}$ & $\lambda_{0} / 4$ & $20-\mathrm{dB}: 0.38$ & 0.3 \\
\hline [29] & $\begin{array}{c}\text { Single-section } \\
\text { Trantanella }\end{array}$ & $\lambda_{0} / 4$ & $20-\mathrm{dB}: 0.36$ & 0.2 \\
\hline [30] & $\begin{array}{c}\text { Single-section } \\
\text { Complex Termination }\end{array}$ & $\lambda_{0} / 4$ & 20-dB: 0.15 & 0.1 \\
\hline [14] & $\begin{array}{l}\text { Complex Isolation } \\
\text { Components }\end{array}$ & $\lambda_{0} / 4$ & 20-dB: 0.10 & 0.15 \\
\hline [21] & $\begin{array}{l}\text { Single-section Modified } \\
\text { Isolation Network }\end{array}$ & $\lambda_{0} / 4$ & 18-dB: 0.40 & 0.5 \\
\hline $\begin{array}{l}\text { This Work } \\
\text { (Fig. 14) }\end{array}$ & $\begin{array}{l}\text { Single-section Optimized } \\
\text { Isolation Network }\end{array}$ & $\lambda_{0} / 4$ & 20-dB: 0.68 & 0.2 \\
\hline $\begin{array}{l}\text { This Work } \\
\text { (Fig. 18) }\end{array}$ & $\begin{array}{l}\text { Single-section Optimized } \\
\text { Isolation Network }\end{array}$ & $\lambda_{0} / 4$ & 19-dB: 0.75 & 0.2 \\
\hline
\end{tabular}

bandwidth, the input return loss drops to $17 \mathrm{~dB}$ at the band edges due to the nonideal effects.

Measured input-output return loss, isolation and insertion loss responses are shown in Figs. 16 and 17. The measurement results match the simulation results well. In the operation band, the extra insertion loss is less than $0.2 \mathrm{~dB}$ and the amplitude mismatch between the output ports is lower than $0.025 \mathrm{~dB}$.

As discussed in (28) of Section III-A, the shorted $\lambda_{0} / 4$ lines at the isolation arm can be replaced with lumped components. For a verification, a modified version of the divider in Fig. 14 is implemented (Fig. 18). Equation (28) results in $L_{p}=23.5 \mathrm{nH}$ and $C_{p}=1.08 \mathrm{pF}$ for $116 \Omega$ characteristic impedance and $1-\mathrm{GHz}$ center frequency. Available chip inductor with a value closest

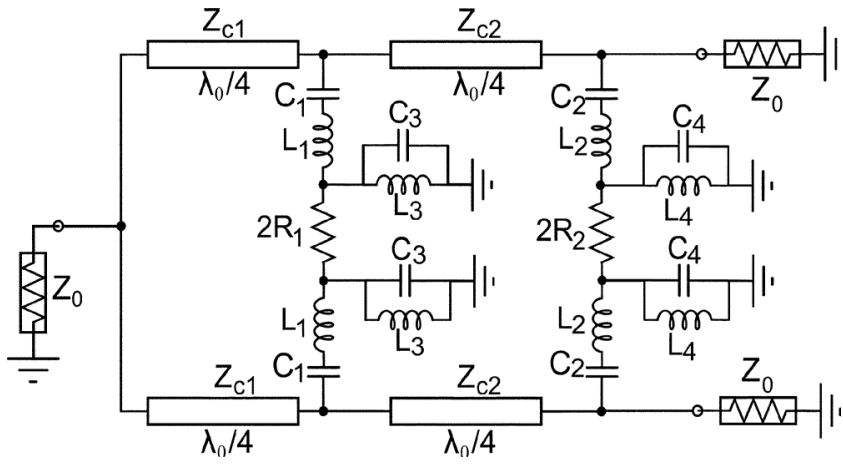

Fig. 21. Two-section divider with the optimal isolation networks.
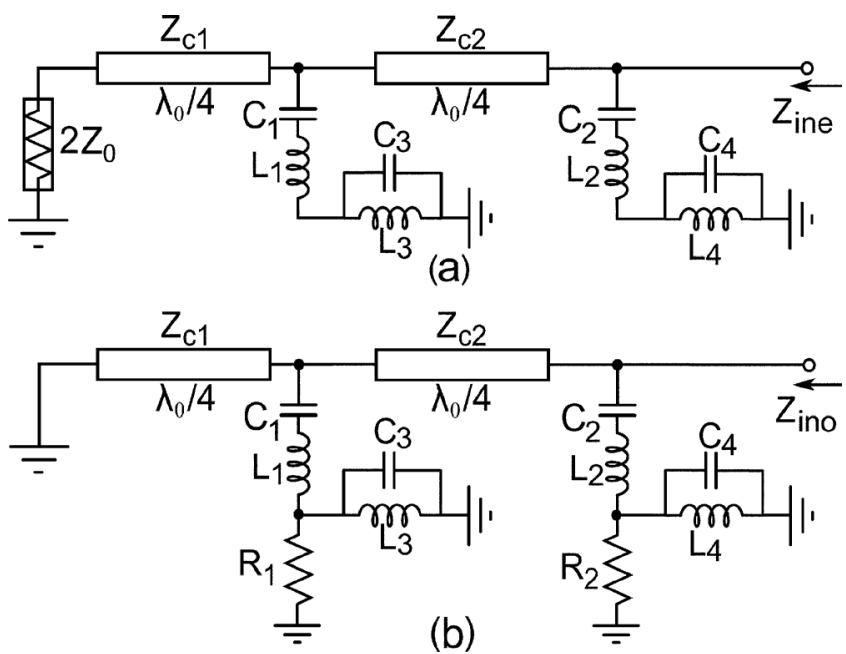

Fig. 22. Even mode (a) and odd mode (b) equivalent circuits of the divider in Fig. 21.

TABLE III

NORMALIZED COMPONENT VALUES FOR INPUT-OUTPUT RETURN LOSSES AND ISOLATION BETTER THAN $\delta(\mathrm{dB})$ FOR THE DiVIDER IN Fig. 21

\begin{tabular}{|c|c|c|c|c|c|c|c|c|}
\hline$\delta$ & $\overline{\Delta f}$ & $\bar{Z}_{c 1}$ & $\bar{Z}_{c 2}$ & ${\overline{w_{0} L}}_{2}$ & ${\overline{w_{0} L_{3}}}$ & ${\overline{w_{0} L}}_{4}$ & $2 \bar{R}_{1}$ & $2 \bar{R}_{2}$ \\
\hline \hline-20 & 1.15 & 1.60 & 1.25 & 0.68 & - & 6.88 & 1.97 & 3.36 \\
\hline-25 & 0.97 & 1.63 & 1.22 & 1.03 & - & 5.84 & 1.76 & 3.76 \\
\hline-30 & 0.80 & 1.66 & 1.21 & 1.30 & 26.8 & 5.30 & 1.74 & 4.25 \\
\hline-35 & 0.65 & 1.67 & 1.20 & 1.53 & 13 & 4.94 & 1.63 & 4.27 \\
\hline-40 & 0.53 & 1.67 & 1.19 & 1.65 & 10 & 4.77 & 1.46 & 3.94 \\
\hline
\end{tabular}

to the calculated one is a 20 -nH 0805 package chip inductor. S-parameter data provided by the manufacturer shows that the equivalent inductance of the 20 -nH chip inductor increases up to $35 \mathrm{nH}$ at $2 \mathrm{GHz}$ due to the parasitic capacitance. This requires a reduction in the calculated value of $C_{p}$. The best performance is obtained using a 0.8 -pF capacitor. Figs. 19 and 20 show the simulated and measured results, respectively. The characteristic is similar to the case with the shorted line. 19-dB bandwidth spans the $0.6 \mathrm{GHz}$ to $1.35 \mathrm{GHz}$ frequency range. The insertion loss is again below $0.2 \mathrm{~dB}$ in the operation bandwidth.

Table II is a comparison ${ }^{5}$ of the dividers of recent studies. Given normalized bandwidth values are valid at the specified

\footnotetext{
${ }^{5}$ The loss comparison in the table is not totally fair, because the upper frequencies and the substrate materials of the dividers listed in the table are not the same.
} 
$\mathrm{dB}$ values of input return loss, output return loss and isolation. Our designs have a wide bandwidth with a low insertion loss and compact size, at the expense of the increased number of components. This increases the circuit implementation complexity and the parasitic problem.

\section{CONCLUSION}

Starting from a fundamental S-parameter relation of a lossless power divider, we find that minimizing the even and odd mode reflection coefficients of an output port is sufficient to optimize a power divider. Using this fact, we are able to synthesize even and odd mode isolation networks to maximize the bandwidth of a single-section Wilkinson divider. The reflection coefficients of the isolation network that perfectly match the even and odd mode circuits are calculated individually. Then, circuits are designed to realize the required reflection coefficients. The even mode and odd mode isolation networks are merged in a configuration to load each other minimally. Analytical expressions are derived for the component values of those networks. A modification in the final component values may be required to alleviate the loading effects. Table I lists the optimal component values.

The resulting divider has a considerably wider operation bandwidth compared to the classical Wilkinson divider. Experimental verification is presented proving that the proposed low-loss divider is promising for wide band applications. The method can be applied to a two-section divider. Using a similar isolation arm for both sections, it is possible to extend the bandwidth considerably.

\section{APPENDIX \\ Two-Section Divider With THE Optimized ISOLATION NETWORKS}

The optimized isolation network is applied to the two-section case as shown in Fig. 21. The even mode and odd mode equivalent circuits are shown in Fig. 22. $Z_{\text {ine }}$ and $Z_{\text {ino }}$ represent the input impedances in each mode, respectively.

At $f_{0}$, the even mode circuit shown in Fig. 22(a) is composed of only $Z_{c 1}$ and $Z_{c 2}$. They provide an impedance transformation from $2 Z_{0}$ to $Z_{0}$. The optimal impedance values satisfy the geometric mean condition [31]:

$$
Z_{c 1} Z_{c 2}=2 Z_{0}^{2}
$$

By allowing a nonzero reflection coefficient magnitude of $\delta$, the bandwidth of operation can be extended. At the band center, $f_{0}$, we have a real reflection coefficient of $\delta[23]$. So, we can write

$$
\frac{Z_{c 2}^{2} 2 Z_{0}}{Z_{c 1}^{2}}=Z_{0} \frac{1+\delta}{1-\delta}
$$

Using (42) and (43), $Z_{c 1}$ and $Z_{c 2}$ are calculated as

$$
\begin{aligned}
& \bar{Z}_{c 1}=\frac{Z_{c 1}}{Z_{0}} \approx 2^{3 / 4}\left(1-\frac{\delta}{2}\right) \\
& \bar{Z}_{c 2}=\frac{Z_{c 2}}{Z_{0}} \approx 2^{1 / 4}\left(1+\frac{\delta}{2}\right) .
\end{aligned}
$$

Even-mode input impedance can be expressed as

$$
\begin{aligned}
& Z_{\text {ine }}=\left(Z_{c 2} \frac{Z_{x}+j Z_{c 2} \tan \left(\frac{\pi f}{2 f_{0}}\right)}{Z_{c 2}+j Z_{x} \tan \left(\frac{\pi f}{2 f_{0}}\right)}\right) \\
& \|\left(j 2 \pi f L_{2}\left(1-\frac{f_{0}^{2}}{f^{2}}\right)+\frac{j 2 \pi f L_{4}}{1-\frac{f_{0}^{2}}{f^{2}}}\right)
\end{aligned}
$$

where

$$
\begin{aligned}
Z_{x}=\left(Z_{c 1} \frac{2 Z_{0}+j Z_{c 1} \tan \left(\frac{\pi f}{2 f_{0}}\right)}{Z_{c 1}+j 2 Z_{0} \tan \left(\frac{\pi f}{2 f_{0}}\right)}\right) \\
\|\left(j 2 \pi f L_{1}\left(1-\frac{f_{0}^{2}}{f^{2}}\right)+\frac{j 2 \pi f L_{3}}{1-\frac{f_{0}^{2}}{f^{2}}}\right) .
\end{aligned}
$$

The odd mode input impedance can be expressed as

$$
\begin{aligned}
Z_{\text {ino }}= & \left(Z_{c 2} \frac{Z_{y}+j Z_{c 2} \tan \left(\frac{\pi f}{2 f_{0}}\right)}{Z_{c 2}+j Z_{y} \tan \left(\frac{\pi f}{2 f_{0}}\right)}\right) \\
& \|\left(j 2 \pi f L_{2}\left(1-\frac{f_{0}^{2}}{f^{2}}\right)+\left(\frac{j 2 \pi f L_{4}}{1-\frac{f_{0}^{2}}{f^{2}}} \| R_{2}\right)\right)
\end{aligned}
$$

where

$$
\begin{aligned}
& Z_{y}=j Z_{c 1} \tan \left(\frac{\pi f}{2 f_{0}}\right) \\
& \|\left(j 2 \pi f L_{1}\left(1-\frac{f_{0}^{2}}{f^{2}}\right)+\left(\frac{j 2 \pi f L_{3}}{1-\frac{f_{0}^{2}}{f^{2}}} \| R_{1}\right)\right) .
\end{aligned}
$$

The optimal values of the remaining components are found by solving (46) and (48) numerically for the maximal bandwidth. The results indicate that the $L_{1}, C_{1}$ pair is not needed. For $20 \mathrm{~dB}$ and $25 \mathrm{~dB}$ cases $L_{3}, C_{3}$ pair is also absent and hence the first isolation network consists of only a resistor, $2 R_{1}$. The normalized component values verified using a microwave circuit simulator are tabulated in Table III. The performance of this divider is depicted in Fig. 13. Its bandwidth is considerably better than that of a two-section Wilkinson divider.

\section{REFERENCES}

[1] E. Wilkinson, "An N-way hybrid power divider," IEEE Trans. Microw. Theory Tech., vol. MTT-8, no. 1, pp. 116-118, Jan. 1960.

[2] S. Cohn, "A class of broadband three-port TEM-mode hybrids," IEEE Trans. Microw. Theory Tech., vol. MTT-19, no. 2, pp. 110-116, Feb. 1968.

[3] R. Ekinge, "A new method of synthesizing matched broad-band TEMmode three-ports," IEEE Trans. Microw. Theory Tech., vol. MTT-19, no. 1, pp. 81-88, Jan. 1971.

[4] H. Oraizi and A.-R. Sharifi, "Design and optimization of broadband asymmetrical multisection Wilkinson power divider," IEEE Trans. Microw. Theory Tech., vol. 54, no. 5, pp. 2220-2231, May 2006.

[5] N. Ehsan, K. Vanhille, S. Rondineau, E. Cullens, and Z. Popovic, "Broadband micro-coaxial Wilkinson dividers," IEEE Trans. Microw. Theory Tech., vol. 57, no. 11, pp. 2783-2789, Nov. 2009.

[6] J.-C. Chieh and A.-V. Pham, "Development of a broadband Wilkinson power combiner on liquid crystal polymer," in Proc. Asia Pacific Microw. Conf., Dec. 2009, pp. 2068-2071. 
[7] J.-S. Lim, U.-H. Park, S. Oh, J. J. Koo, Y.-C. Jeong, and D. Ahn, “A 800 to 3200-mhz wideband cpw balun using multistage Wilkinson structure," in Proc. IEEE MTT-S Int. Microw. Symp. Dig., Jun. 2006, pp. 1141-1144.

[8] S. Wong and L. Zhu, "Ultra-wideband power dividers with good isolation and improved sharp roll-off skirt," IET Microw. Antennas Propag., vol. 3, no. 8, pp. 1157-1163, Dec. 2009.

[9] P. Goodman, "A wideband stripline matched power divider," in Proc. G-MTT Int. Microwave Symp., May 1968, pp. 16-20.

[10] C. T. Chiang and B.-K. Chaung, "Ultra wideband power divider using tapered line," Progress In Electromagnetics Res, vol. 106, pp. 61-73, 2010.

[11] K. Hettak, G. Morin, and M. Stubbs, "Compact mmic cpw and asymmetric cps branch-line couplers and Wilkinson dividers using shunt and series stub loading," IEEE Trans. Microw. Theory Tech., vol. 53, no. 5, pp. 1624-1635, May 2005.

[12] R. Mirzavand, M. Honari, A. Abdipour, and G. Moradi, "Compact microstrip Wilkinson power dividers with harmonic suppression and arbitrary power division ratios," IEEE Trans. Microw. Theory Tech., vol. 61, no. 1, pp. 61-68, Jan. 2013.

[13] Y.-S. Lin and J.-H. Lee, "Miniature ultra-wideband power divider using bridged t-coils," IEEE Microw. Compon. Lett., vol. 22, no. 8, pp. 391-393, Aug. 2012.

[14] X. Wang, I. Sakagami, A. Mase, and M. Ichimura, "Wilkinson power divider with complex isolation component and its miniaturization," IEEE Trans. Microw. Theory Tech., vol. 62, no. 3, pp. 422-430, Mar. 2014.

[15] T. Kawai, J. Yamasaki, Y. Kokubo, and I. Ohta, "A design method of dual-frequency Wilkinson power divider," in Proc. Asia Pacific Microw. Conf., 2006, pp. 913-916.

[16] X. Wang and I. Sakagami, "Generalized dual-frequency Wilkinson power dividers with a series/parallel RLC circuit," in Proc. IEEE MTT-S Int. Microw. Symp. Dig., Jun. 2011, pp. 1-4.

[17] Y. Wu, Y. Liu, and S. Li, "Unequal dual-frequency Wilkinson power divider including series resistor-inductor-capacitor isolation structure,' IET Microw. Antennas Propag., vol. 3, no. 7, pp. 1079-1085, 2009.

[18] Y. Wu, Y. Liu, and Q. Xue, "An analytical approach for a novel coupled-line dual-band Wilkinson power divider," IEEE Trans. Microw. Theory Tech., vol. 59, no. 2, pp. 286-294, Feb. 2011.

[19] Y. Wu, Y. Liu, Y. Zhang, J. Gao, and H. Zhou, "A dual band unequal Wilkinson power divider without reactive components," IEEE Trans. Microw. Theory Tech., vol. 57, no. 1, pp. 216-222, Jan. 2009

[20] A. Genc and R. Baktur, "Dual-and triple-band Wilkinson power dividers based on composite right-and left-handed transmission lines," IEEE Trans. Compon. Packag. Manuf. Technol., vol. 1, no. 3, pp. 327-334, Mar. 2011.

[21] J.-C. Kao, Z.-M. Tsai, K.-Y. Lin, and H. Wang, "A modified Wilkinson power divider with isolation bandwidth improvement," IEEE Trans. Microw. Theory Tech., vol. 60, no. 9, pp. 2768-2780, Sep. 2012.

[22] S.-F. Chao and Y.-R Li, "Miniature filtering power divider with increased isolation bandwidth," Electron. Lett., vol. 50, no. 8, pp. 608-610, Apr. 2014.

[23] V. Tas and A. Atalar, "A performance enhanced power divider structure," in IEEE MTT-S Int. Microw. Symp. Dig., 2014, pp. 1-4.

[24] D. M. Pozar, Microwave Engineering. New York, NY, USA: Wiley, 1998.

[25] S. Horst, R. Bairavasubramanian, M. Tentzeris, and J. Papapolymerou, "Modified Wilkinson power dividers for millimeter-wave integrated circuits," IEEE Trans. Microw. Theory Tech., vol. 55, no. 11, pp. 2439-2446, Nov. 2007.
[26] C. Trantanella, "A novel power divider with enhanced physical and electrical port isolation," in IEEE MTT-S Int. Microw. Symp. Dig., May 2010, pp. 129-132.

[27] X. Lan, P. Chang-Chien, F. Fong, D. Eaves, X. Zeng, and M. Kinits, "Ultra-wideband power divider using multi-wafer packaging technology," IEEE Microw. Compon. Lett., vol. 21, no. 1, pp. 46-48, Jan. 2011.

[28] S.-H. Kim, J.-H. Yoon, Y. Kim, and Y.-C. Yoon, "A modified Wilkinson divider using zero-degree phase shifting composite right/left-handed transmission line," in IEEE MTT-S Int. Microw. Symp. Dig., 2010, pp. 1556-1559.

[29] X. wang, I. Sakagami, A. Mase, and M. Ichimura, "Trantanella Wilkinson power divider with additional transmission lines for simple layout," IET Microw. Antennas Propag., vol. 8, no. 9, pp. 666-672, Jun. 2014.

[30] H. R. Ahn and S. Nam, "3-dB power dividers with equal complex termination impedances and design methods for controlling isolation circuits," IEEE Trans. Microw. Theory Tech., vol. 61, no. 11, pp. 3872-3883, Nov. 2013.

[31] S. B. Cohn, "Optimum design of stepped transmission-line transformers," IRE Trans. Microw. Theory Tech., vol. MTT-3, no. 3, pp 16-20, Apr. 1955

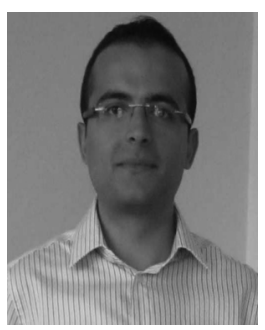

Vahdettin Tas (S'14) received the B.S. degree from Middle East Technical University, Ankara, Turkey, in 2007 and the M.S. degree from Bilkent University, Ankara, Turkey, in 2009, both in electrical engineering, where he is currently pursuing the Ph.D. degree.

In 2009, he was with the Electrical Engineering and Computer Science Department, University of California, Berkeley, CA, USA, as a Research Assistant. From 2010 to 2011, he was an RF Design Engineer at Meteksan Defense, Inc. Since 2011, he has been with the Power Amplifier Technologies Divison, Aselsan, Inc. His current research interests include broadband and efficient power amplification, power coupling, and power combining techniques.

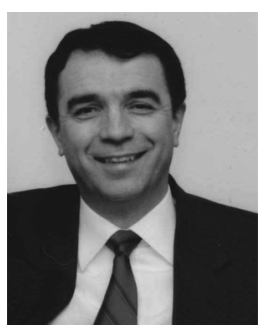

Abdullah Atalar (M'88-SM'90-F'07) received the B.S. degree from Middle East Technical University, Ankara, Turkey, in 1974, and the M.S. and Ph.D. degrees from Stanford University, Stanford, CA, USA in 1976 and 1978, respectively, all in electrical engineering.

He was with Hewlett Packard Labs, Palo Alto, CA, USA, in 1979. From 1980 to 1986 , he was on the faculty of the Middle East Technical University as an Assistant Professor. In 1986, he joined the Bilkent University as the chairman of the Electrical and Electronics Engineering Department and served in the founding of the Department where he is currently a Professor. In 1995, he was a Visiting Professor of Stanford University. From 1996 to 2010, he was the Provost of Bilkent University. $\mathrm{He}$ is currently the Rector of the same university. His current research interests include micromachined devices and microwave electronics. He was awarded the Science Award of TUBITAK in 1994. 